Purdue University Purdue e-Pubs

Weldon School of Biomedical Engineering Faculty

Publications

Weldon School of Biomedical Engineering

1985

\title{
Joseph S. Redding's Contributions to Cardiac Resuscitation
}

Sandra H. Ralston

Charles F. Babbs

Follow this and additional works at: http://docs.lib.purdue.edu/bmepubs

Part of the Biomedical Engineering and Bioengineering Commons

This document has been made available through Purdue e-Pubs, a service of the Purdue University Libraries. Please contact epubs@purdue.edu for additional information. 


\title{
Joseph S. Redding's Contributions to Cardiac Resuscitation
}

\author{
Sandra H. Ralston, RN, PhD \\ Charles F. Babbs, MD, PhD \\ Biomedical Engineering Center \\ Purdue University
}

American Journal of Emergency Medicine 1985, Volume 3, Number 3, Pages 247-251

The experimental studies conducted by the late Joseph S. Redding over the past three decades with his students and colleagues form the scientific basis for many aspects of present cardiopulmonary resuscitation (CPR). Redding's research was characterized by its elegance, simplicity, common-sense reasoning, and clinical relevance. Much of his work conducted in the 1960's anticipated with uncanny accuracy some of the most actively discussed issues in resuscitation today (Table 1). Most interesting are Redding's classic studies related to diastolic arterial pressure and myocardial perfusion during CPR, abdominal binding, acid-base status, and use of methoxamine versus other adrenergic drugs for cardiac resuscitation. Al though he trained and matured as a researcher before the specialty of emergency medicine existed; his work addressed many important topics of current academic interest in this specialty. Here, we review nine areas of Joseph S. Redding's research in hope that readers will find his life's work to be a guide and inspiration in carrying on the search for improved resuscitation methods in the clinic and in the laboratory.

\section{CLOSED- VERSUS OPEN-CHEST CARDIAC MASSAGE}

Cardiopulmonary resuscitation, as it is practiced today, was reintroduced in 1960 when Kouwenhoven et al. ${ }^{1}$ published their benchmark paper on closed-chest cardiac massage. The technique was developed in a series of over $100 \mathrm{dogs}$ and then applied clinically, resulting in the survival of 14 of the first 20 patients. Before then, the treatment of cardiac-arrest victims required opening the chest and directly massaging the heart to produce an artificial circulation. By combining closed-chest cardiac compression with the techniques of mouth to nose ventilation and closed-chest defibrillation already developed, resuscitation could be initiated immediately by anyone, with no special equipment. 
With healthy skepticism, Redding first decided to find out how the new closed-chest procedure compared with the old open-chest method. ${ }^{2}$ Beginning with pilot studies conducted on over 40 dogs, Redding developed the techniques he would use before the actual experiments were conducted. As there were no standards at the time for performing CPR, Redding developed his own, based on clinical and animal experience. He preferred interposing ventilations between chest compressions because evaluating chest expansions and the possibility of airway obstruction was nearly impossible when the compressions and ventilations occurred simultaneously. He also demonstrated the dangers of trauma to the chest wall, heart, and liver that resulted when maximal force was used on the chest to obtain the highest possible arterial pressures. His research team opted for a more moderate chest-compression force, which they found would facilitate successful resuscitation while avoiding the trauma. ${ }^{2}$ The importance of optimizing chest compression force in order to obtain satisfactory results with closed chest CPR has only recently been rediscovered. 3

After techniques had been optimized in preliminary studies, 20 dogs were subjected to 20 minutes of either closed-chest massage or thoracotomy with direct cardiac massage during ventricular fibrillation (VF). All 20 animals were successfully resuscitated after defibrillation. This study confirmed the value of closed- chest massage and demonstrated that it was as effective as the open-chest method in restoring circulation. In addition, the team observed and reported the pros and cons of the open- and closed-chest techniques. They found that the open-chest technique could be initiated rapidly. Itrequired only 25 to 52 seconds from VF to massage in these studies, which compared favorably with the immediate onset closedchest method. However, the open-chest method had inherent disadvantages. The necessity of performing a thoracotomy added insult to an already compromised victim and limited its application.

In addition to the obvious detrimental effects of open-chest massage, Redding found that the open-chest method was far more fatiguing to the operator's arms than the closed-chest method, necessitating more than one resuscitator for each 20 -minute period. ${ }^{2}$ As survival following arrest was possible with both methods, the subtle additional advantages suggested a superiority of the closed-chest technique. 
TABLE 1. Joseph S. Redding's Contributions to Cardiopulmonary Resuscitation (CPR)

Documentation of the efficacy of closed-chest versus open-chest CPR (1961)

Reporting of dangers of excessive chest compression force (1961)

Development of an animal model of cardiac arrest (1962)

Definition of indications for specific resuscitative measures (1962)

Establishment of the value of epinephrine therapy (1963)

Promotion of early epinephrine therapy (1963)

Promotion of high-dose epinephrine therapy (1963)

Definition of the importance of peripheral vascular tone (1965)

Definition of diastolic arterial pressure during CPR necessary for survival $(1965,1971)$

Evaluation of mechanical devices for closed-chest CPR (1966)

Definition of the effective routes for drug administration during CPR (1967)

Definition of guidelines for sodium bicarbonate therapy (1967)

Description of an arteriovenous pH difference during CPR (1967)

Warning against indiscriminate use of sodium bicarbonate (1967)

Comparison of standard CPR with simultaneous ventilation-compression CPR using manual techniques (1981)

Promotion of the use of methoxamine for electromechanical dissociation (1983)

\section{ANIMAL MODEL OF CARDIAC ARREST WITH ELECTROMECHANICAL DISSOCIATION}

To investigate these newly defined resuscitative measures, Redding and Pearson ${ }^{4}$ developed an asphyxial model of cardiac arrest adapted from an earlier study done by Schwann et al. ${ }^{5}$ They hypothesized that this particular model, leading to what we would now identify as electromechanical dissociation (EMD), paralleled many clinical situations of cardiac arrest. For example, unconsciousness resulting from any cause frequently leads to upper airway obstruction and, ultimately, to cardiac arrest. Moreover, as Redding and Pearson pointed out, whenever the circulation ceases, "resuscitation from myocardial anoxia is a preliminary to re-establishment of the circulation."

Their experimental protocol, applied to 80 anesthetized dogs, began when the endotracheal tube was clamped at the end of an expiration. Progressive stages of asphyxia were carefully recorded. At first, the animals showed intense efforts to breathe accompanied by bradycardia and blood pressure fluctuations. After 2-4 minutes, these efforts ceased, and hypotension and bradycardia increased. After 6-8 minutes of airway obstruction aortic pressure approached zero and systolic fluctuations ceased, although electrocardiographic complexes typically persisted. 
To identify which resuscitative measures were indicated and effective in the various stages of asphyxia, the animals were divided into 8 groups. They were subjected to varying degrees of asphyxia ranging from brief airway obstruction to 10 minutes of complete circulatory arrest. The results from these studies not only defined an animal model for studying resuscitation procedures, but also defined guidelines for the type of resuscitation procedure to be used. Specifically, during the early stages of asphyxia (2-4 minutes) when the circulation was good, ventilation alone produced recovery. However, when the systolic blood pressure dropped below $100 \mathrm{~mm} \mathrm{Hg}$, ventilation was inadequate in restoring the circulation. Redding advised: "With a doubtful circulation, closed-chest cardiac massage is needed as well as ventilation ... When a pulse can barely be felt, or is absent, we feel that closed-chest cardiac massage must be started immediately."

\section{EPINEPHRINE IN RESUSCITATION}

Redding was quite aware of the limitations of the artificial circulation provided by external chest compressions. His studies showed that it was only possible to create about $15-20 \%$ of the normal carotid blood flow by these means alone, resulting in restoration of the circulation in only a few animals. ${ }^{2-4}$ In an attempt to improve the success of closed-chest resuscitation, he decided to investigate the use of adjunctive drugs. Epinephrine was a logical first choice, because this drug had been used to treat cardiac arrest victims throughout the century. ${ }^{6}$ With both alpha- and beta-adrenergic actions, epinephrine could both constrict the peripheral vasculature, thereby raising the central arterial pressure, and stimulate inotropic and chronotropic activity of the heart. Its use was controversial, however, because of its capacity to produce cardiac excitability that sometimes resulted in ventricular fibrillation. To determine whether the use of epinephrine was warranted in the treatment of either asystole or fibrillation, Redding injected $1 \mathrm{mg}$ of epinephrine into the ventricular chambers of dogs subjected to varying degrees of asphyxial arrest. ${ }^{7-8}$ The results of these studies showed that when ventilation and closed-chest cardiac massage were started 1 minute after circulatory arrest, only two of ten animals were resuscitated. However, when intracardiac epinephrine was added to these resuscitation measures, the circulation was restored in nine of ten animals after a 10-minute arrest period. Redding concluded that "the danger of ventricular fibrillation following the use of epinephrine in the anoxic myocardium should no longer contraindicate its use in cases in which an electrocardiograph and external defibrillator are available.

The value of epinephrine therapy was thereby firmly established, and Redding included some additional, perhaps obvious, advice to insure that the drug was administered correctly. He warned that in the presence of circulatory arrest, one must accompany the administration of epinephrine with cardiac massage to distribute the drug to its site of action. In a later study, he identified this site as peripheral systemic arterioles, but then he speculated that the site could be either the myocardium or the peripheral vascular system. 
Pearson and Redding ${ }^{7}$ proved the value of giving epinephrine early in resuscitation by performing the following experiment. Using their asphyxial model, two groups of anesthetized dogs were subjected to 2 minutes of circulatory arrest. After beginning CPR, intracardiac epinephrine ( $1 \mathrm{mg}$ ) was given either 1 minute or 10 minutes later. Both short- and long-term recovery were better in the group receiving epinephrine early in the resuscitation regime. The message was clear: "Since the likelihood of re-establishing circulation decreases as time passes after circulatory arrest, injection of epinephrine should not be delayed in order to obtain electrocardiographic differentiation.

Although the literature indicated a wide range of possible epinephrine doses, Redding empirically found that $1 \mathrm{mg}$ would work satisfactorily in nearly all patients, including children, during arrest. Very small infants were given $0.1 \mathrm{mg}$ increments that were repeated as necessary. ${ }^{8}$ Administering an epinephrine dose that would work was of prime importance to Redding, who believed that the "treatment of cardiac arrest was no time for homeopathic doses. "

\section{PERIPHERAL VASCULAR TONE}

There was initial speculation that epinephrine's value in resuscitation was because of its action on the myocardium that improved both inotropy and chronotropy during electromechanical dissociation (EMD) and asystole, or because of its ability to coarsen fbrillation, an action thought (but never proven) to improve chances of defibrillation. These beliefs were dispelled when Redding tested a variety of different drugs during resuscitation in the animal model. Interestingly, he found that the pure alpha agonists phenylephrine, metaraminol, and methoxamine with only peripheral vasoconstrictor action were always as effective as epinephrine in restoring the circulation. However, drugs that directly stimulated the myocardium were never so effective as the vasoconstrictors in restoring the circulation. In fact, the use of isoproterenol, a pure beta agonist, resulted in no survivors, an outcome worse than that in the saline-treated control group. A subsequent study by Yakaitis et al ${ }^{10}$ (Yakaitis was Redding's former student) obtained no better results with the somewhat selective $\beta_{1}$ agonist, dobutamine. Redding repeatedly tested the use of calcium chloride incardiac arrest and found it to be inferior to epinephrine in restoring the circulation. ${ }^{11}$ Late in his career he compared the use of calcium chloride with that of methoxamine in the treatment of asphyxial EMD and found calcium chloride to be far less effective. ${ }^{12}$ These studies may figure importantly in the revision of American Heart Association standards for the use of calcium chloride in cardiac resuscitation.

Redding concluded that drugs with only cardiac stimulating capabilities are of no value in resuscitation from cardiac arrest and that calcium chloride, in particular, should be avoided because of its possibly harmful effects. ${ }^{12}$ He further concluded that the value of epinephrine was not in its direct action on the heart, but rather in its action on the peripheral vasculature. More recently, studies by Yakaitis and coworkers ${ }^{10}$ have 
confirmed Redding's studies. Prior to experimental asphyxial arrest, dogs were pre-treated with drugs producing either alpha- or beta-receptor blockade. Resuscitation was instituted following a 5-minute arrest period, and either alpha- or beta-adrenergic drugs were systematically tested. These studies showed that alpha blockade, but not beta blockade, significantly decreased the incidence of survival following cardiac arrest and CPR. $10-13$

\section{DIASTOLIC ARTERIAL PRESSURE}

In 1906, Crile and Dolley reported that resuscitation success was closely related to achieving a diastolic arterial pressure of $30-40 \mathrm{~mm} \mathrm{Hg}$. They indicated that these pressures were nearly impossible to achieve with only closed-chest massage and ventilations and that the addition of epinephrine was required. Redding's work solidly confirmed these early studies. He found that very few animals receiving CPR alone were resuscitated. ${ }^{4,11,12}$ However, when the peripheral resistance was increased using either vasoconstrictor drugs or abdominal binding, there was an increase in the diastolic arterial pressure and in the successful return of the circulation. ${ }^{14}$ In this study, Redding found "complete correlation between return of spontaneous circulation and the development of amlic diastolic arterial pressures above $40 \mathrm{~mm} \mathrm{Hg}$, as well as complete correlation between failure of resuscitation and aortic diastolic pressures below 40 mm Hg." (Recent experiments have suggested a most plausible mechanism: the strong correlation of myocardial perfusion during CPR with the diastolic arterial pressure and diastolic arteriovenous pressure difference. ${ }^{15}$ )

\section{OPTIMUM ROUTES FOR DRUG ADMINISTRATION}

As a result of Redding's studies, the importance of combining prompt vasoconstrictor drug therapy with life-support measures was clearly established and accepted by the mid-1960's. However, the most effective route for drug administration remained a controversial subject. Some physicians were reluctant to use an intravenous route even when it was readily available and instead favored the intracardiac route. However, intracardiac injections could lead to unfavorable post- arrest complications, such as pneumothorax or he mopericardium. The intracardiac route also necessitated interruption of chest compressions, perhaps for considerable periods of time if the cardiac ventricles were difficult to locate. To study the relative effectiveness of various drug routes. Redding again used the asphyxial model in 80 anesthetized dogs. ${ }^{16}$ After 5 minutes of circulatory arrest and 1 minute of CPR, epinephrine was given via one of the selected routes. The intramuscular, intravenous, and intracardiac routes were obvious sites for investigation, but the team also included the intratracheal route in their study.

The knowledge that certain drugs could be absorbed into the circulation through the membranes of the trachea and bronchi was well established by this time, but the method had not actually been employed clinically. Redding reasoned that an endotracheal tube that could be quickly and easily inserted by the resuscitation team would provide an airway 
and access to the systemic circulation. The study proved the intra-muscular routes (both into the tongue and thigh muscles) were of no benefit, probably because of the poor circulation that would result in poor absorption from the muscles. Undiluted epinephrine $(1 \mathrm{mg} / \mathrm{ml})$ given intratracheally was also of no benefit in restoring the circulation. However, the authors reported "...that intratracheal (diluted), intravenous, and intracardiac routes are equally effective, and that whichever of these routes is most immediately available should be used."

\section{GUIDELINES FOR BICARBONATE THERAPY}

During the early 1960's, sodium bicarbonate became a popular adjunctive drug for use with CPR. Its use was advocated to correct the lactic acidosis known to accompany circulatory arrest and anaerobic metabolism. There was additional evidence to suggest that epinephrine was not so effective during severe acidosis and that animals pre-treated with bicarbonate maintained a normal $\mathrm{pH}$ level and were easier to resuscitate. Redding observed that excessively large amounts of bicarbonate were being used and that often bicarbonate was preferred to drugs already proven effective, such as epinephrine. Therefore, to determine the usefulness of sodium bicarbonate in resuscitation, 85 anesthetized dogs were studied after 10 minutes of circulatory arrest caused by asphyxia. They were given bicarbonate, epinephrine, or a combination of these drugs at the onset of CPR. ${ }^{17}$ Sodium bicarbonate therapy proved to be no better than therapy using no drug, while epinephrine $(1 \mathrm{mg})$ restored the circulation in 16 of 20 animals. This result was significantly better than eight of 20 animals in the bicarbonate group.

To determine whether the use of bicarbonate might potentiate the effects of epinephrine, a sub-optimum dose of $0.2 \mathrm{mg}$ of epinephrine was combined with $4.4 \mathrm{mEq} / \mathrm{kg}$ of sodium bicarbonate. This combination was no more effective in restoring the circulation than epinephrine $(0.2 \mathrm{mg})$ alone. These results all indicated that, although bicarbonate was not harmful, an adequate dose of epinephrine was far superior. In both asphyxial and ventricular fibrillation models, a combination of high-dose epinephrine and bicarbonate proved to be even better than either of these agents alone. ${ }^{18}$ The results of these studies led Redding to recommend that "sodium bicarbonate may be given with, or following adequate doses of epinephrine to restore the circulation."

\section{ARTERIOVENOUS pH GAP}

Arterial blood samples are the usual sources for $p H$ determinations, and many physicians advocate their use during CPR to provide guidelines for bicarbonate administration. In a small group of animals, Redding measured both arterial and venous $\mathrm{pH}$ during $\mathrm{CPR}$ and made some surprising observations. ${ }^{17}$ At the onset of CPR, there was a rapid rise in $p H$ that declined over time. However, during the resuscitation period, the arterial $p H$ was far more alkalemic as compared with the venous $p H$ producing a large arteriovenous $p H$ difference. He attributed this gap to the poor tissue perfusion that allowed for metabolic acid accumulation on the venous side. When bicarbonate was given to these animals, the $p H$ approximated normal if 
the circulation was restored. When the circulation was not restored, the bicarbonate administration resulted in severe arterial alkalemia. The key implications of these findings were "that accurate correction of metabolic acidosis during circulatory arrest is impractical prior to restoration of spontaneous circulation." Sub- sequent studies have confirmed these findings. ${ }^{19}$ Indeed, the indiscriminate use of bicarbonate may be detrimental not only in producing iatrogenic alkalosis, but also by increasing plasma osmolality, factors that may decrease the patient's chances for survival. ${ }^{2}$

\section{METHOXAMINE}

The use of epinephrine during resuscitation from cardiac arrest was controversial before the mid-1960's. Now its use is again being questioned. Recent studies show that epinephrine's powerful beta-adrenergic stimulation, by increasing the vigor of fibrillation, increases the oxygen demand and may in part cancel the benefits derived from its valuable enhancement of myocardial blood flow. ${ }^{21-22}$ Although Redding's work clearly demonstrated the value of epinephrine, it also showed that alpha agonists were equally effective. Possibly one of the vasoconstrictor drugs without myocardial stimulating capacity could be more effective than epinephrine in difficult resuscitations.

In an earlier study, Redding showed that the circulation was restored in twice as many methoxamine treated dogs than epinephrine-treated dogs after 10 minutes of fibrillation. However, the combination of epinephrine and bicarbonate was comparable with methoxamine in restoring the circulation, and it resulted in significantly more long term (24 hour) survival. Redding reasoned that the longer-acting vasoconstrictor activity of methoxamine might have contributed to a metabolic acidosis incompatible with life. He also reasoned that this could have been averted with post-resuscitation intensive care and that methoxamine plus bicarbonate might have been superior to epinephrine plus bicarbonate.

Still active in 1983, Redding compared methoxamine with drugs currently recommended for the treatment of electromechanical dissociation. Again using his asphyxial arrest model, Redding and coworkers found that methoxamine was far more effective than either calcium chloride or atropine in promoting the return of the circulation after 5 minutes of circulatory arrest. Despite its potential problems, Redding strongly recommended that methoxamine be "the drug of choice during attempted resuscitation from cardiac arrest regardless of the ECG pattern." ${ }^{12}$

\section{CONCLUSIONS}

Through his work, Joseph Redding will live for years to come. While other might eventually have made all of the same discoveries, Redding was quick to define the crucial questions in the early days of CPR and was quite effective in finding answers. His career is a sterling example of the harmonious combination of meticulously acquired laboratory data, extensive clinical experience, and clear reasoning. His papers are still required reading today for clinicians and 
scientists endeavoring to establish an academic tradition in emergency medicine.

\section{REFERENCES}

1. Kouwenhoven WB, Jude JR, Knickerbocker GG. Closed- chest cardiac massage. JAMA 1960;173:1064-1067.

2. Redding JS, Cozine RA. A comparison of open-chest and closed-chest cardiac massage in dogs. Anesthesiology $1961 ; 22: 280-285$.

3. Babbs CF, Voorhees WD, Fitzgerald KR, et al. Relationship of artificial cardiac output to chest compression amplitude--Evidence for an effective compression threshold. Ann Emerg Med 1983;12:527-532.

4. Redding JS, Pearson JW . Resuscitation from asphyxia. JAMA 1962;182:283-286.

5. Schwann HG, Brucer M. Sequence of circulatory, respiratory, and cerebral failure during process of death--Its relation to resuscibility. Tex Rep Bioi Med 1951;9:180219.

6. Crile G, Dolley DT. Experimental research into resuscitation of dogs - killed by anesthetics and asphyxia. J Exp Med 1906;8:713-720.

7. Pearson JW, Redding JS. Epinephrine in cardiac resuscitation. Am Heart J 1963;66:210-214.

8. Pearson JW, Redding JS. The role of epinephrine in cardiac resuscitation. Anesth Analg 1963;42:599-606.

9. Redding JS. Cardiopulmonary resuscitation: An algorithm and some common pitfalls. Am HeartJ 1979;98:788-797.

10. Yakaitis RW, Otto CW, Blitt CD. Relative importance of alpha and beta adrenergic receptors during resuscitation. Grit Care Med 1979;7:293.

11. Redding JS, Pearson JW. Evaluation of drugs for cardiac resuscitation. Anesthesiology 1963;24:203-207.

12. Redding JS, Haynes RR, Thomas JD. Drug Therapy in resuscitation from electromechanical dissociation. Crit Care Med 1983;11:681-684.

13. Otto CW, Yakaitis RW, Blitt CD. Mechanism of action of epinephrine in resuscitation from asphyxial arrest. Grit Care Med 1981;9:364-365.

14. Redding JS. Abdominal compression in cardiopulmonary resuscitation. Anesth Analg $1971 ; 50: 668-675$.

15. Ralston SH, Voorhees WD, Babbs CF. Intrapulmonary epinephrine during cardiopulmonary resuscitation: lmproved regional blood flow and resuscitation in dogs. Ann Intern Med 1984;13:79-86.

16. Redding JS, Asuncion JS, Pearson JW. Effective routes of drug administration during cardiac arrest. Anesth Analg 1967;46:253-258.

17. Redding JS, Pearson JW. Metabolic acidosis: A factor in cardiac resuscitation. South Med J 1967;60:926-932.

18. Redding JS, Pearson JW. Resuscitation from ventricular fibrillation. JAMA $1968 ; 203: 255-260$.

19. Ralston SH, Voorhees, WD, Showen L, et al. Venous and arterial blood gases during and after cardiopulmonary resuscitation in dogs. Am J Emerg Med 1985;3:132-136.

20. Weil MH. Iatrogenic alkalosis in CPR. Emerg Med 1981;13:55-63. 
21. Livesay JL, Follette OM, Fey KH, et al. Optimizing myocardial supply/demand balance with alpha-adrenergic drugs during cardiopulmonary resuscitation. J Thorac Cardiovasc Surg 1978;76:244-251.

22. Ditchey RV. High-dose epinephrine does not improve the balance between myocardial oxygen supply and demand during cardiopulmonary resuscitation in dogs. J Am Coli Cardiol 1984;3:596. 